\title{
Perfil epidemiológico dos casos de tuberculose no estado do Maranhão de 2009 a 2018
}

\author{
Epidemiological profile of tuberculosis cases in the state of Maranhão from 2009 to 2018 \\ Perfil epidemiológico de los casos de tuberculosis en el estado de Maranhão de 2009 a \\ 2018
}

Amadeu Rodrigues Passarinho Neto ${ }^{1}$, Kécya Patricia Costa Macêdo ${ }^{1}$, Jéssica Larissa Sousa Vaz ${ }^{2}$, Soliane Cristina Rodrigues Costa ${ }^{2}$, Sarah Daisy Mota Feitosa ${ }^{2}$, Viviane Cardoso Neves ${ }^{2}$, Roseane Mara Cardoso Lima Verde ${ }^{3}$, Matheus Hipólito do Nascimento ${ }^{4}$, Leonardo Ferreira Soares ${ }^{5}$, Francisco das Chagas Araújo Sousa ${ }^{6}$, Arionildo Batista Moraes ${ }^{7}$, Evaldo Hipólito de Oliveira*

\section{RESUMO}

Objetivo: Delinear o perfil epidemiológico dos casos de tuberculose notificados no Estado do Maranhão entre os anos de 2009 e 2018. Métodos: Estudo de natureza retrospectiva, descritiva e quantitativa sobre as ocorrências de tuberculose notificadas no estado do Maranhão, delineando seu perfil epidemiológico. Resultados: Os resultados obtidos no estudo revelam que no período de 2009 a 2018 foram registradas 23.120 notificações de caso de Tuberculose no Estado do Maranhão, onde se encontra atualmente em 4ํㅜㄴ lugar na região Nordeste e em 11ำ no país. Conclusão: $O$ estudo demonstrou que mesmo com a grande quantidade do número de casos de Tuberculose no Estado do Maranhão, houve uma grande diminuição do número de pessoas infectadas ao longo do período analisado. Foi demonstrado que a doença acomete mais as pessoas do sexo masculino e, entre os 20 aos 39 anos de idade.

Palavras-chave: Mycobacterium tuberculosis, Tuberculose. Maranhão, Tratamento.

\begin{abstract}
Objective: Outline the epidemiological profile of tuberculosis cases reported in the State of Maranhão between 2009 and 2018. Methods: Retrospective, descriptive and quantitative study on the occurrences of tuberculosis reported in the state of Maranhão, outlining its epidemiological profile. Results: The results obtained in this study reveal that in the period from 2009 to 2018, 23.120 notifications of tuberculosis cases were registered in the State of Maranhão, where it is currently ranked 4th in the Northeast region and 11th in the country. Conclusion: The study showed that even with the large number of tuberculosis cases in the State of Maranhão there was a large decrease in the number of people infected over the period analyzed. It has been shown that the disease affects more males, between 20 and 39 years of age.
\end{abstract}

Key words: Mycobacterium tuberculosis, Tuberculosis, Maranhão, Treatment.

\section{RESUMEN}

Objetivo: Delinear el perfil epidemiológico de los casos de tuberculosis notificados en el estado de Maranhão entre 2009 y 2018. Métodos: Estudio retrospectivo, descriptivo y cuantitativo sobre los casos de tuberculosis notificadas en el estado de Maranhão, que describe su perfil epidemiológico. Resultados: Los resultados obtenidos en el estudio revelan que, en el período de 2009 a 2018, se registraron 23.120 notificaciones de caso de Tuberculosis en el Estado de Maranhão, donde se encuentra actualmente en 4임 luar en la región

${ }^{1}$ Centro Universitário Santo Agostinho. Teresina - PI.

2Universidade Federal do Piaú. Curso de Farmácia. Teresina - PI. *E-mail: evaldohipolito@gmail.com

${ }^{3}$ Programa de Pós-Graduação em Engenharia Biomédica - Universidade Brasil. São Paulo - SP.

${ }^{4}$ Faculdade de Odontologia. Centro Universitário Unieuro. Brasília - DF.

${ }^{5}$ Universidade Estadual da Paraíba. João Pessoa - PB.

6Universidade Estadual do Piaú. Faculdade de Ciências Médicas. Teresina - PI.

${ }^{7}$ Centro Universitário UNINOVAFAPI. Teresina - PI.

SUBMETIDO EM: 5/2019

ACEITO EM: 7/2019

PUBLICADO EM: 8/2020

REAS/EJCH | Vol.Sup.n.53 | e992 | DOI: https://doi.org/10.25248/reas.e992.2020 Página 1 de 11 
Nordeste y en el $11^{\circ}$ en el país. Conclusión: El estudio mostró que incluso con la gran cantidad del número de casos de Tuberculosis en el Estado de Maranhão hubo una gran disminución del número de personas infectadas a lo largo del período analizado. Se ha demostrado que la enfermedad afecta a más varones, entre los 20 y los 39 años de edad.

Palabras clave: Mycobacterium tuberculosis, La tuberculosis, Maranhão, Tratamiento.

\section{INTRODUÇÃO}

A tuberculose (TB) é uma patologia causada pelo bacilo álcool ácido resistente (BAAR) Mycobacterium tuberculosis, sendo a principal espécie patogênica do gênero Mycobacterium (SANTOS J, 2007). Ela é transmitida por inoculação, ingestão de alimentos contaminados ou inalação por via aérea de partículas infectantes suspensas no ar através de um indivíduo contaminado a outro sadio (PILLER RV, 2012).

Considerada como infectocontagiosa, caracteriza-se por um longo período de latência e por ocorrer preferencialmente nos pulmões, apesar de, em alguns casos, ser verificada em outros órgãos, como ossos, rins e meninges e provocar resposta granulomatosa com inflamação e lesão tissular (RUFFINO-NETTO A, 2002). A TB pode levar à morte, porém pode ser tratada atingindo a cura do paciente. É preciso tomar certas medidas, pois a bactéria possui a capacidade de ser transmitida de pessoa a pessoa (BERALDO AA, et al., 2012).

A não continuidade do tratamento tem sido apontado por diversos estudos como um grave problema que leva a manutenção da cadeia de transmissão do bacilo, em virtude que o paciente não tratado adequadamente continua como fonte de infecção, aumentando o risco de agravamento da doença, de mortalidade e favorecendo o desenvolvimento de bactérias resistentes aos fármacos, dificultando o monitoramento da patologia (SAN PEDRO A e OLIVEIRA RMD, 2003). No Brasil, a TB é um sério problema da saúde pública, com profundas raízes sociais. Progressivamente por ano, são notificados aproximadamente 70 mil novos quadros e cerca de 4,5 mil mortes em sua decorrência (BARREIRA D e GRANGEIRO A, 2007). A doença se expressa mais existente nas regiões cujas esferas socioeconômicas da população são mais deficientes (MENDES ADM e FENSTERSEIFER LM, 2004).

A utilização de técnicas de geoprocessamento como ferramenta de prevenção, vigilância o manejo da infecção por Mycobacterium tuberculosis constitui-se como um importante instrumento de gestão em saúde capaz de contribuir para o organismo e avaliação das ações de saúde, de forma a mapear, monitorar e disseminar as informações em saúde no âmbito do Sistema Único de Saúde (SUS) propiciando a evolução do sistema de saúde como um todo, em especial a Atenção Primária (AP) à Saúde Pública, por intermédio de recursos que proporcionarão melhoria da qualidade da informação nesse setor (CHIRINOS NEC, MEIRELLES BHS, 2011). O comportamento da tuberculose, como de outras endemias, é fortemente influenciado pelo meio, sendo possível evidenciar sua associação com precárias condições socioeconômicas, reiterando, portanto, a necessidade de estudá-la para arquitetar as intervenções adequadas (PONCE MAZ, et al., 2013). Diante disso, o estudo teve como objetivo delinear o perfil epidemiológico dos casos de infecção por $M$. tuberculosis notificados no Estado do Maranhão entre os anos de 2009 e 2018.

\section{MÉTODOS}

Estudo de natureza retrospectiva, descritiva e quantitativa sobre os casos confirmados de TB notificados no Estado do Maranhão, entre os anos de 2009 e 2018, delineando o perfil epidemiológico deste estado. As informações foram apuradas durante os meses de fevereiro e março de 2019, utilizando como fonte o Sistema de Informação de Agravos de Notificação (SINAN), base de dados disponibilizada pelo Departamento de Informática do Sistema Único de Saúde (DATASUS). As variáveis estudadas foram: ano de notificação, município de notificação, zona de residência, sexo, raça, faixa etária, escolaridade, tipo de entrada, forma clínica, doenças associadas (Síndrome da Imunodeficiência Adquirida - SIDA, alcoolismo, diabetes, doença mental, tabagismo, drogas ilícitas, outras doenças), $1^{\circ}$ e $2^{\circ}$ baciloscopia de escarro, cultura de escarro e situação de encerramento. 
Para os cálculos de incidência acessaram-se, por meio da plataforma do DATASUS, as estimativas de população residente calculadas pelo Instituto Brasileiro de Geografia e Estatística (IBGE) e o Sistema de Informações sobre Mortalidade (SIM). A taxa de incidência (I) da TB na população em determinado ano foi obtida multiplicando-se o quociente entre o número de casos e a população residente por cem mil, obtendose o número de casos de TB a cada 100 mil habitantes.

$$
I=\frac{\text { Número de casos }}{\text { População residente }} \times 100.000
$$

A taxa de mortalidade (M) foi obtida de forma semelhante, multiplicando-se o quociente entre o número de óbitos por TB e o número de óbitos no estado por cem mil.

$$
M=\frac{\text { Número de óbitos }}{\text { População residente }} \times 100.000
$$

O tratamento dos dados coletados foi realizado por meio do software Microsoft Excel® 2013 e, para melhor análise da disposição dos casos pelo estado, utilizou-se o programa Tab para Windows - TabWin, versão 4.14 .

\section{RESULTADOS}

No intervalo compreendido entre os anos de 2009 e 2018 foram registradas 23.120 notificações de casos de pacientes tuberculosos no Estado do Maranhão, caracterizando-o como $4^{\circ}$ estado da região nordeste com maior número de notificações e $11^{\circ}$ do país. Na figura 1, observa-se a distribuição destes casos pelo estado. Dos 217 municípios, a capital São Luís foi a maior notificadora com 9.566 registros (41,37\%); seguida de Imperatriz, 723 ocorrências (3,18\%) e Caxias, 617 casos (2,67\%), como demonstra o mapa de saúde (Figura 1).

Figura 1 - Distribuição dos casos confirmados de tuberculose notificados no Estado do Maranhão, entre os anos de 2009 e 2018.

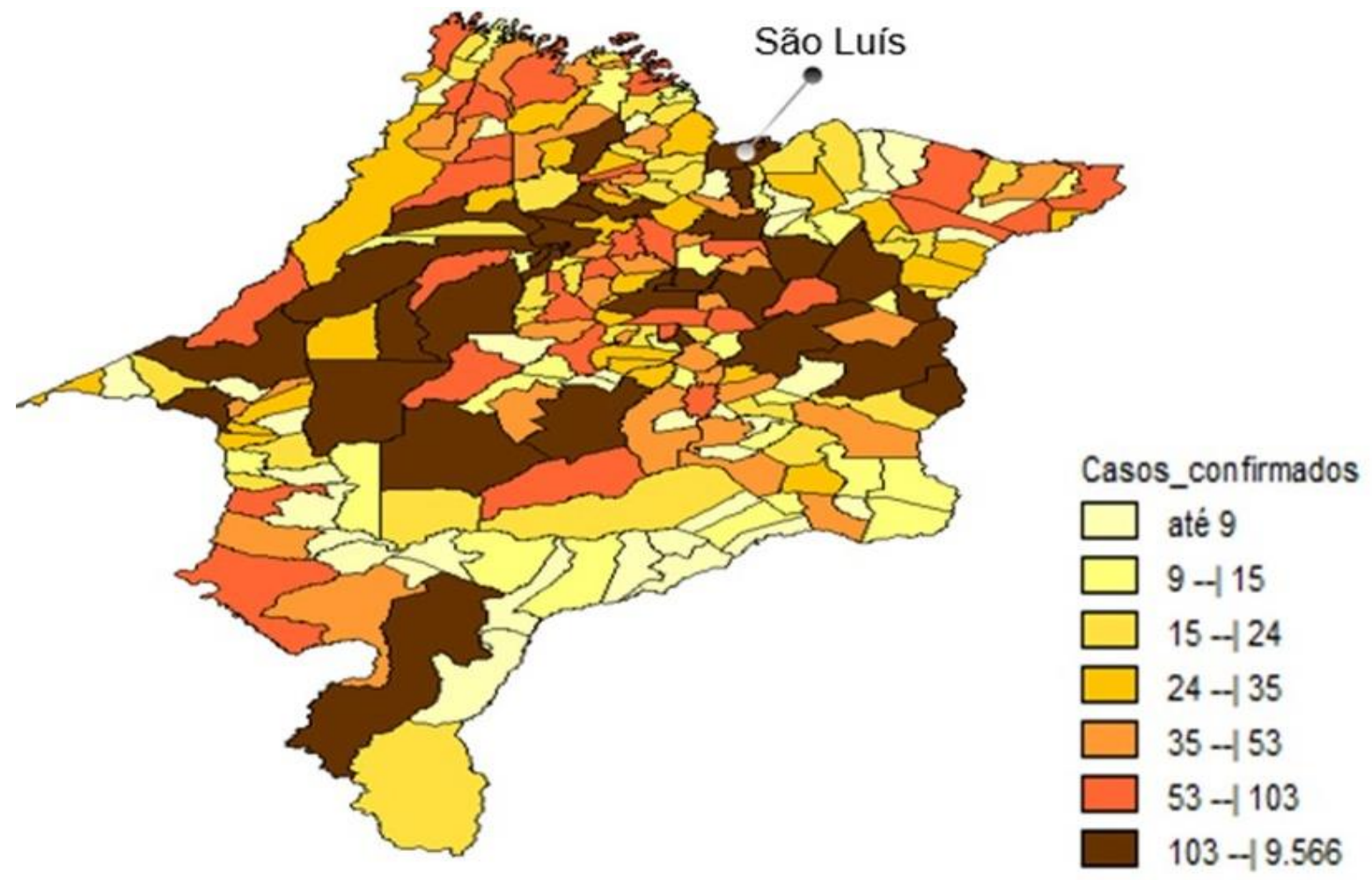

Fonte: Neto ARP, et al., 2020. Baseado em: Ministério da Saúde/SVS/Sinan Net, 2020. 
A maior fração dos casos de TB compreende pacientes que vivem na área urbana do estado, 17.000 registros $(70,37 \%$ da população residente). Enquanto 6.396 casos referem-se a pacientes da zona rural $(26,47 \%), 261$ a zona periurbana (1,08\%) e em 499 registros essa informação foi ignorada $(2,06 \%)$.

No Gráfico 1 é possível observar a flutuação do número de notificações durante uma década e sua tendência geral a diminuição, verificando-se que, de 2009 a 2018, houve uma diminuição de $24,81 \%$ no número de notificações por ano. Além disso, determinou-se o índice de incidência da doença no estado, constatando-se que está abaixo da observada para a região nordeste (26/100 mil hab.) e para o país (28,15/100 mil hab.) no ano de 2018 e que ao longo dos 10 anos analisados os índices de incidência no estado sempre seguiu as tendências observadas na região e no país.

Gráfico 1 - Taxa de incidência e número de casos confirmados de tuberculose notificados no Estado do Maranhão no período de 2009 a 2018, segundo ano de notificação.

$\square$ Casos confirmados $\longrightarrow$ Taxa de incidência (x 100 mil hab.)

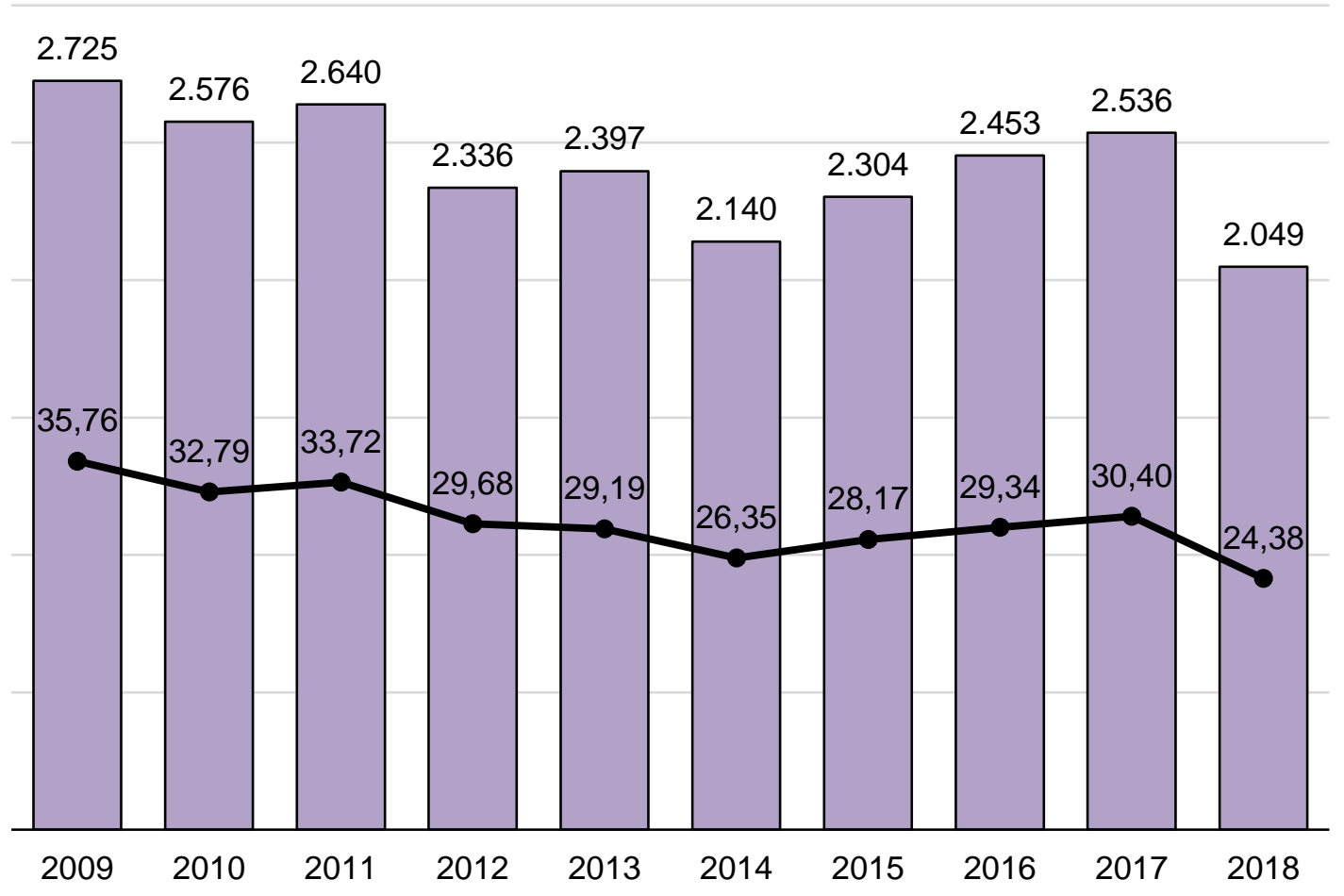

\section{Ano de notificação}

Fonte: Neto ARP, et al., 2020. Baseado em: Ministério da Saúde/SVS - Sinan Net; IBGE - Estimativas para o TCU, 2020.

As características sociodemográficas da população com TB foram resumidas na Tabela 1, onde se pode notar a alta prevalência em pessoas do sexo masculino (64,03\%) e naquelas de raça parda $(68,96 \%)$. Em relação à faixa etária, observou-se que ao longo dos anos analisados o intervalo com maior incidência de casos foi o de 20 a 39 anos (42,72\%) seguido da faixa de $40-59$ anos de idade $(29,22 \%)$. Finalmente, a menor quantidade de casos concentra-se entre os pacientes com ensino superior completo $(2,29 \%)$ e incompleto $(1,68 \%)$. 
Tabela 1 - Casos confirmados de tuberculose notificados no Estado do Maranhão entre os anos de 2009 e 2018 segundo variáveis sociodemográficas.

\begin{tabular}{|c|c|c|}
\hline \multirow{2}{*}{ Variável } & \multicolumn{2}{|c|}{ Frequência } \\
\hline & $\mathbf{n}$ & $\%$ \\
\hline \multicolumn{3}{|l|}{ Sexo } \\
\hline Feminino & 8.689 & 35,97 \\
\hline Masculino & 15.467 & 64,03 \\
\hline \multicolumn{3}{|l|}{ Raça } \\
\hline Ign/Branco & 352 & 1,46 \\
\hline Branca & 3.156 & 13,07 \\
\hline Preta & 3.278 & 13,57 \\
\hline Amarela & 175 & 0,72 \\
\hline Parda & 16.659 & 68,96 \\
\hline Indígena & 536 & 2,22 \\
\hline \multicolumn{3}{|l|}{ Faixa etária } \\
\hline Em branco & 9 & 0,04 \\
\hline$<1$ ano & 182 & 0,75 \\
\hline $01-04$ & 134 & 0,55 \\
\hline $05-09$ & 141 & 0,58 \\
\hline $10-14$ & 343 & 1,42 \\
\hline $15-19$ & 1.510 & 6,25 \\
\hline $20-39$ & 10.320 & 42,72 \\
\hline $40-59$ & 7.058 & 29,22 \\
\hline $60-64$ & 1.201 & 4,97 \\
\hline $65-69$ & 1.044 & 4,32 \\
\hline $70-79$ & 1.571 & 6,50 \\
\hline$>80$ anos & 643 & 2,66 \\
\hline \multicolumn{3}{|l|}{ Escolaridade } \\
\hline Ign/Branco & 2.330 & 9,65 \\
\hline Analfabeto & 3.175 & 13,14 \\
\hline 1a a $4^{a}$ série incompleta do EF & 4.641 & 19,21 \\
\hline $4^{a}$ série completa do EF & 1.976 & 8,18 \\
\hline $5^{\circ}$ a 8ª série incompleta do EF & 3.770 & 15,61 \\
\hline EF completo & 1.498 & 6,20 \\
\hline EM incompleto & 1.819 & 7,53 \\
\hline EM completo & 3.602 & 14,91 \\
\hline ES incompleta & 405 & 1,68 \\
\hline ES completa & 552 & 2,29 \\
\hline Não se aplica & 388 & 1,61 \\
\hline
\end{tabular}

Legenda: Ign (Ignorado); EF (Ensino Fundamental); EM (Ensino Médio); ES (Ensino Superior).

Fonte: Neto ARP, et al., 2020. Baseado em: Ministério da Saúde/SVS/Sinan Net, 2020.

No Gráfico 2 pode-se analisar a distribuição dos casos confirmados de TB de acordo com o tipo de entrada. No tocante ao número de casos novos de TB por ano, calculou-se uma redução de $38,60 \%$ de 2009 para 2018, porcentagem maior do que o observado para a região nordeste $(31,41 \%)$ e para o país $(20,86 \%)$, no mesmo período. Além disso, determinou-se um aumento de 45,6\%, de 2009 para 2018, na taxa de reingresso após abandono, enquanto na região nordeste esta taxa foi de $27,15 \%$ e no país, $31,48 \%$. 
Gráfico 2 - Distribuição dos casos confirmados de tuberculose notificados no Estado do Maranhão entre os anos de 2009 e 2018, conforme o tipo de entrada.

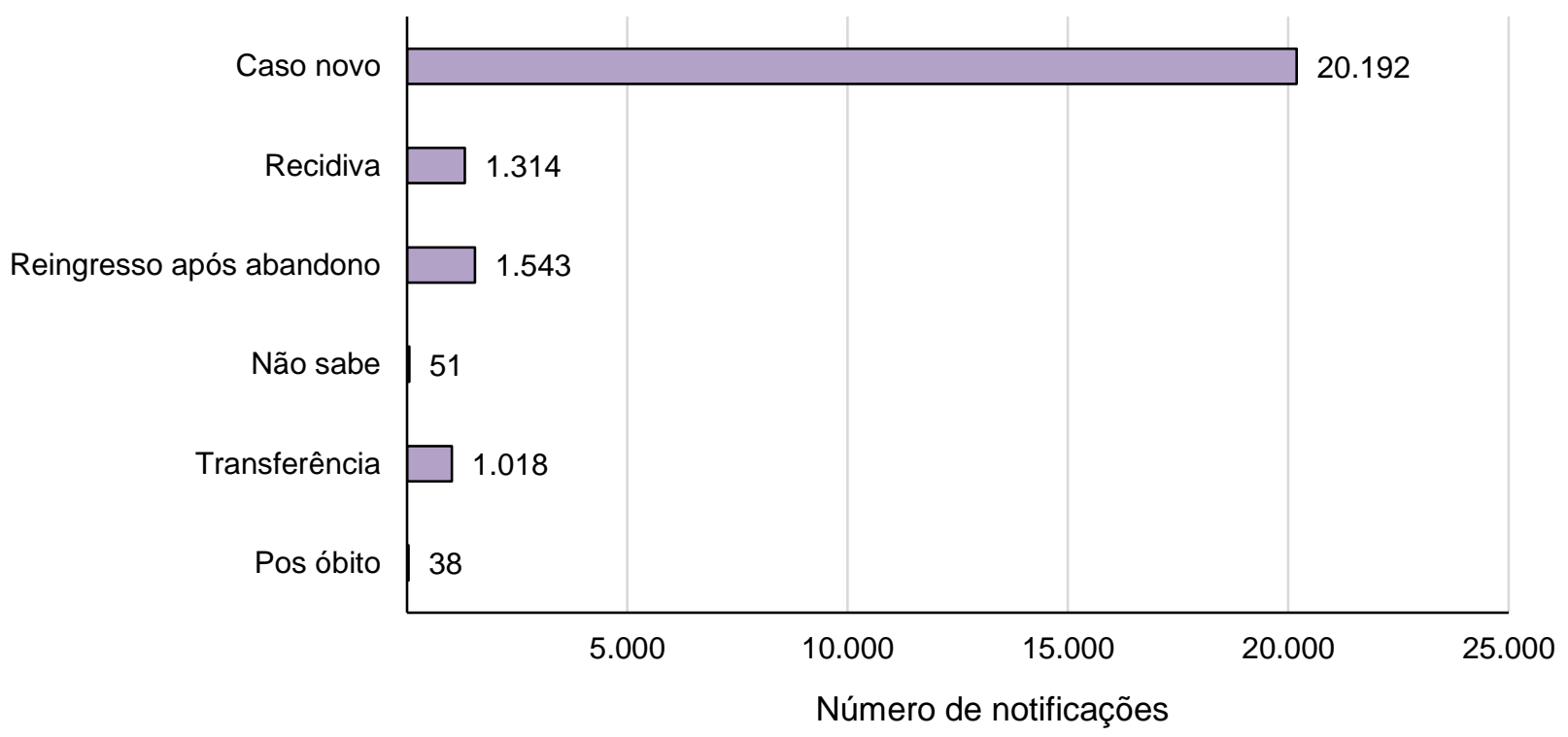

Fonte: Neto ARP, et al., 2020. Baseado em: Ministério da Saúde/SVS/Sinan Net, 2020.

O Gráfico 3 demonstra o número de casos confirmados de TB notificados conforme a forma clínica, podendo ser observado que foram apresentadas apenas duas formas da doença e a sua associação. $O$ tipo pulmonar destaca-se como a maior parte da patologia apresentando 21.541 casos (89\%), seguido da forma extrapulmonar com 2.398 casos (10\%) e a associação pulmonar/extrapulmonar com apenas 212 casos (1\%).

Gráfico 3 - Casos confirmados de Tuberculose notificados no Estado do Maranhão entre os anos de 2009 e 2018, segundo a forma clínica.

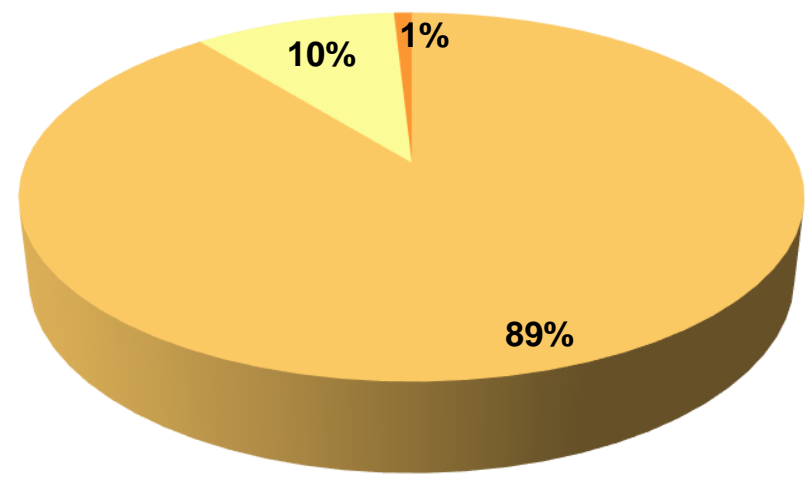

Pulmonar Extrapulmonar $\quad$ Pulmonar + Extrapulmonar

Fonte: Neto ARP, et al., 2020. Baseado em: Ministério da Saúde/SVS/Sinan Net, 2020.

Dentre os exames para a TB realizados para o diagnóstico, o Gráfico 4 apresenta os dados para $1^{\underline{a}}$ e $2^{\underline{a}}$ baciloscopia em comparação com a cultura de escarro, demonstrando que a $1^{\underline{a}}$ e $2^{\underline{a}}$ baciloscopia apresentaram resultados positivos superiores (12.456 e 5.883 casos) em relação a cultura (1.801 casos). Adicionalmente a cultura de escarro apresentou 20.438 casos notificados em que o método não foi realizado. Além disso, apenas a $2^{\underline{a}}$ baciloscopia apresentou 10.592 casos com ignorados/branco. 
Gráfico 4 - Distribuição dos casos confirmados de tuberculose notificados no Estado do Maranhão entre os anos de 2009 e 2018, de acordo $1^{\mathrm{a}}$ e $2^{\mathrm{a}}$ baciloscopia e cultura de escarro.

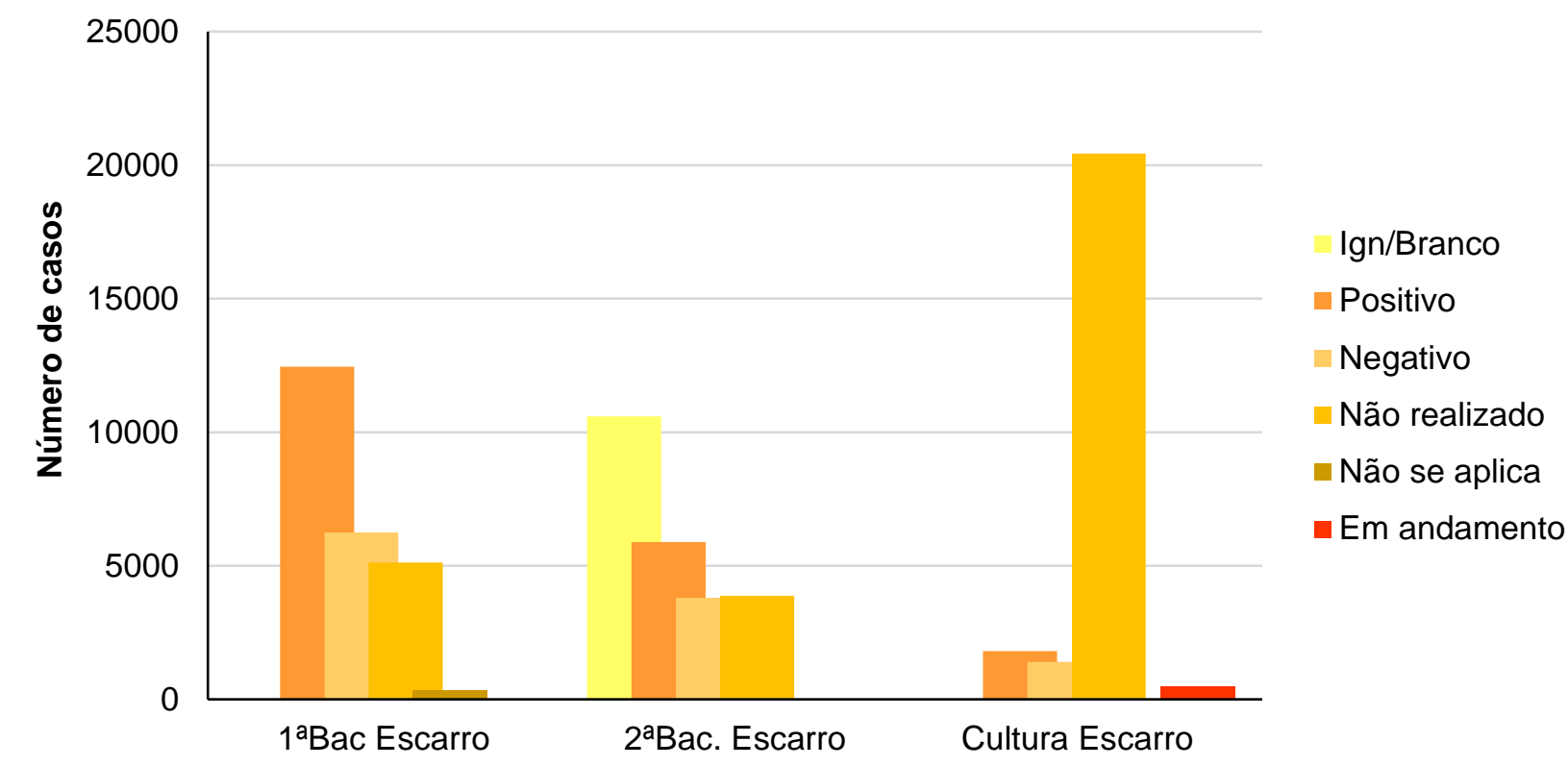

Legenda: Ign. (Ignorado); Bac. (Baciloscopia).

Fonte: Neto ARP, et al., 2020. Baseado em: Ministério da Saúde/SVS/Sinan Net, 2020.

O Gráfico 5 apresenta a distribuição dos casos confirmados notificados de TB em relação a associação com outras morbidades relacionadas ao seu adoecimento. É possível observar que entre os anos de 2009 e 2018 o tabagismo aumentou em 13,29\% e as drogas ilícitas em 4,35\%. Já o alcoolismo sempre obteve um elevado número de casos (2.620 casos no total) que caíram apenas nos anos de 2013 e 2014. Ademais, para SIDA, diabetes, doença mental e outras doenças houve uma flutuação entre elevação e queda nos casos nessa variação de tempo, onde ambas as patologias decaíram no último ano.

Gráfico 5 - Casos confirmados de tuberculose notificados no Estado do Maranhão entre os anos de 2009 e 2018 , segundo as doenças associadas.

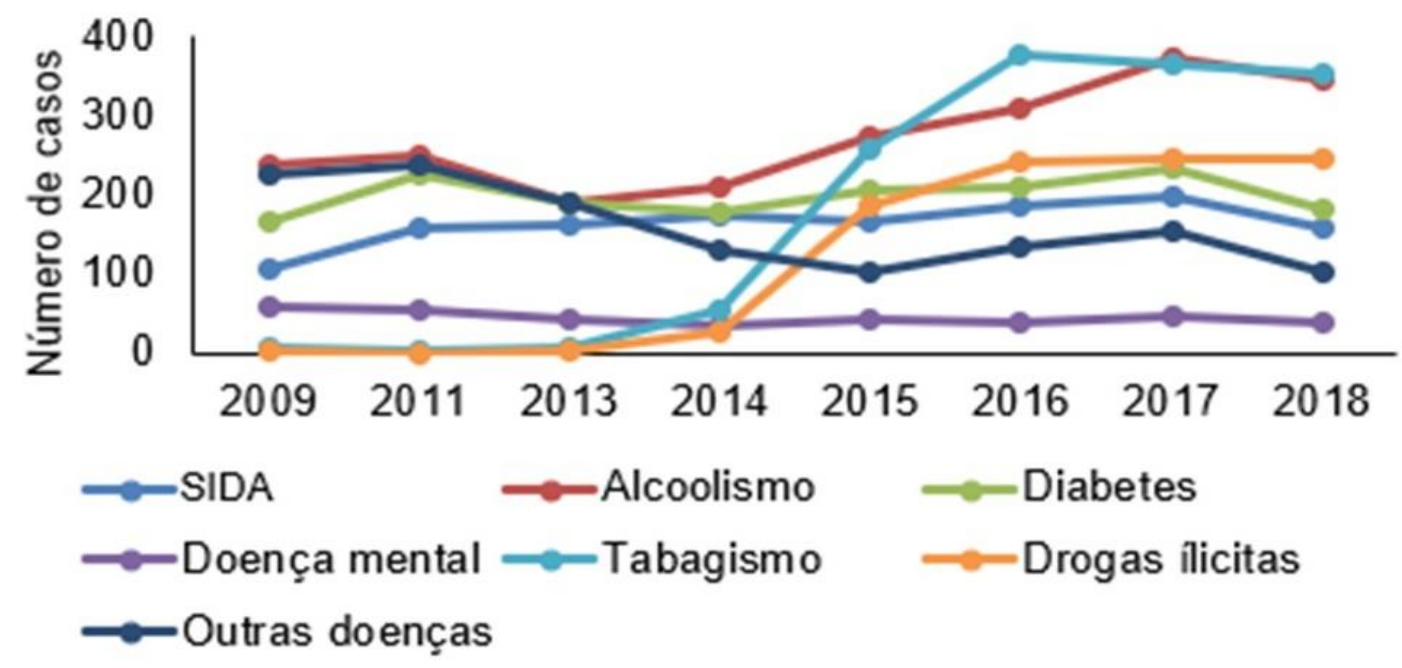

Fonte: Neto ARP, et al., 2020. Baseado em: Ministério da Saúde/SVS/Sinan Net, 2020. 
A Tabela 2 apresenta a situação de encerramento para os casos confirmados de TB, onde entre os pacientes com a doença 15.842 casos $(65,58 \%)$ obtiveram a cura. Todavia, $11,04 \%$ abandonaram 0 tratamento e outros $8,87 \%$ casos foram de transferências. Ademais, identificou-se que em 789 casos houve óbito por outras causas, sendo esse dado superior aos óbitos por TB (782 casos). Em relação à taxa de mortalidade, a maior delas foi registrada em 2012 (38,14 óbitos a cada 100.000 habitantes), observando-se uma redução na mortalidade de 2009 (29,92/100 mil hab.) para 2017 (21,89/100 mil hab.).

Tabela 2 - Casos confirmados de tuberculose notificados no Estado do Maranhão entre os anos 2009 e 2018, conforme situação de encerramento.

\begin{tabular}{lcc}
\hline \multirow{2}{*}{ Situação de encerramento } & \multicolumn{2}{c}{ Frequência } \\
\cline { 2 - 3 } & $\mathbf{N}$ & $\%$ \\
\hline Ign/Branco & 1.660 & 6,87 \\
Cura & 15.842 & 65,58 \\
Abandono & 2.666 & 11,04 \\
Óbito por tuberculose & 782 & 3,24 \\
Óbito por outras causas & 789 & 3,27 \\
Transferência & 2.142 & 8,87 \\
TB-DR & 180 & 0,75 \\
Mudança de Esquema & 32 & 0,13 \\
Falência & 11 & 0,05 \\
Abandono Primário & 52 & 0,22 \\
\hline
\end{tabular}

Legenda: Ign (Ignorado); TB-DR (Tuberculose resistente a medicamentos).

Fonte: Neto ARP, et al., 2020. Baseado em: Ministério da Saúde/SVS/Sinan Net, 2020.

\section{DISCUSSÃO}

O DATASUS existe desde 1991 e tem como competência gerir os sistemas de informação do SUS que são necessários ao processo de planejamento, operação e controle. Atualmente é responsável por apresentar elucidações de software para as secretarias estaduais e municipais de saúde de acordo com as necessidades de seus gestores (DATASUS, 2018).

Conseguimos obter dados específicos sobre as zonas com maior fração de ocorrências, na qual 70,37\% estão estabelecidas na área urbana do Estado, onde segundo a literatura, as condições socioeconômicas são mais precárias, educação deficiente, aglomerado populacional, maior número de má nutrição e alcoolismo, além de experimentar serviços de saúde pouco resolutivos e com limitações em termos de oferta e de elenco de serviços, pois mesmo que várias cidades do estado tenham crescido com planejamento prévio, resultou ainda em áreas consideradas subnormais, classificadas como carentes de serviços públicos e essenciais em sua maioria, portanto consideradas com maior iniquidade social (CHIRINOS NEC, et al., 2013).

Fazendo um comparativo entre os gráficos, é suma importância identificar um declínio do número de casos durante uma década, verificando uma diminuição de $24,81 \%$ do número de notificações por ano, constando que a média está abaixo estabelecida para a região Nordeste. Segundo Grosch CA, et al. (2016), O Ministério da Saúde iniciou no Maranhão visitas de monitoramento e avaliação aos municípios prioritários no controle da tuberculose, adicionalmente a utilização dos dados no SINAN permite o acompanhamento do problema, corroborando para a identificação de aspectos relevantes e incentivando a busca de novas intervenções para o controle da doença.

No Estado do Maranhão houve maior predominância de TB no sexo masculino com $64,03 \%$, pode estar relacionado ao fato de reterem maior consumo de bebidas alcoólicas, o tabagismo e a baixa procura aos serviços de saúde homens (PILLER RV, 2007). Em relação a raça, sobrepõe-se a parda com 68,96\%, que apresentou um crescimento de $43,13 \%$ em 2011, que corresponde a 82,2 milhões, considerada a raça de maior aumento nos últimos 10 anos (CEINFO, 2011). 
A faixa etária com maior prevalência de casos ficou entre os 20 - 39 anos $(42,72 \%)$, supostamente por serem os indivíduos mais evidenciados as condições de risco, por fim os pacientes que apresentam menor quantidade de casos são os pacientes com curso superior completo com 2,29\% e 1,68\% para incompleto.

De acordo o DATASUS obtivemos alguns valores de referência o qual comparados a literatura encontrada em Barreira D e Grangeiro A (2007) mostram que se obteve uma redução no número de casos novos por TB por ano, calculando em $38,60 \%$ de 2009 para 2018 , onde a porcentagem estabelecida para a região Nordeste é de $31,41 \%$ e em todo o país $20,86 \%$ durante o período analisado.

É mostrado também que grande parte da população que possui a doença reestabeleceram a utilização dos medicamentos para o tratamento, obtendo um percentual de $45,6 \%$ no Estado do Maranhão, enquanto na região nordeste a taxa é de $27,15 \%$ e no país de $31,48 \%$. Nesse sentido, esses dados demonstram que ainda há necessidade de aprimoramento e compreensão das razões que levam a persistência da tuberculose como um importante problema de saúde pública no País, de maneira que esses dados sejam elementos para impulsionar o debate e qualificar as ações de redução do impacto dessa patologia (BARREIRA $D$ e GRANGEIRO A, 2007).

A não continuidade do tratamento pode implicar em impactos negativos na contenção da doença e a predisposição a ocorrência de TB multirresistente, condição clinicamente mais severa e com manejo terapêutico mais difícil e dispendioso (BIERRENBACH AL, et al., 2007).

No local do estudo identificou-se duas apresentações da doença- a pulmonar e extrapulmonar. A infecção pulmonar é a mais frequente entre a população, acometendo $89 \%$ dos casos, ou seja, a patologia apresenta em torno de 21.541 casos de pacientes infectados. A infecção pelo $M$. tuberculosis ocorre quando os bacilos atingem com sucesso uma via aérea terminal, resultando em um processo inflamatório pulmonar localizado chamado de foco parenquimatoso ou foco de Ghon (PONCE MAZ, et al., 2013).

Os bacilos disseminam através do sistema linfático para os linfonodos regionais. A forma extrapulmonar acomete 2.398 casos (10\%) e a associação entre pulmonar /extrapulmonar apenas apresentam 212 casos (1\%). Por isso, a forma pulmonar ainda é a de mais fácil diagnostico, o que corrobora com outros estudos (HINO P, 2011; SILVA IP, et al., 2016).

A baciloscopia é um método complementar mais realizado no diagnóstico da TB por ser um exame rápido e acessível economicamente (BARREIRA D e GRANGEIRO A, 2007). Os dados apresentados segundo o DATASUS comparando a baciloscopia com a cultura de escarro apresentou que a $1^{\circ}$ e a $2^{\circ}$ baciloscopia obteve resultado positivo superior a 12.456 e 5.883 casos, respectivamente, e a cultura somente 1.801 casos. $\mathrm{Na}$ tabela obtém dados referentes a cultura de escarro, onde 20.438 casos o método não foi realizado, além da 2 o baciloscopia que apresentou 10.592 casos como ignorados/brancos.

Portanto, o diagnóstico precoce da tuberculose e o início do tratamento são necessários para interromper a evolução da doença, ou seja, melhorando o quadro clínico com repercussões positivas no prognóstico. Desse modo, mesmo a baciloscopia do escarro representando o principal recurso diagnóstico por seu baixo custo e sua simplicidade, suas limitações ainda demandam a realização da cultura do escarro (NOGUEIRA et al., 2004). Além disso, a ausência de informações no sistema de informação pode vir a ocasionar a subnotificação dos casos, bem como gerar um levantamento de dados inadequados, refletindo em intervenções distintas do quadro real de necessidade da população (NETO MS, et al., 2012).

No presente estudo as estatísticas nos mostram quem além da doença TB, a infecção é associada a outros meles da sociedade, como pessoas vivendo com HIV/Aids, causando um aumento no número de casos ou até agravando a situação em que o paciente se encontra. Temos um problema maior que é o alcoolismo onde detém de um elevado número de casos, constando 2.620 no total com percentagem de aumento de $13,29 \%$, seguindo de drogas ilícitas com 4,35\% (BENTO J, et al., 2011).

Já outras doenças como SIDA, diabetes, distúrbios psiquiátricos, houve uma variação de elevação e queda nos últimos anos, devido o maior cuidado intensivo que esses pacientes recebem em unidades terciarias de saúde para o tratamento (CLEMENTINO FDS, et al., 2011). 
Com relação a distribuição espacial da doença e com dados atualizados do sistema informatizado, tivemos com estudo relacionado de uma década que pacientes avaliados durante esse tempo 15.842 casos obtiveram a cura, ou seja, $65,58 \%$ do total, $11,04 \%$ por algum motivo tiveram desistência do tratamento e outros $8,87 \%$ por transferência. Acrescenta-se um número de óbitos por outras causas com 789 , esse número sendo maior que a quantidade de óbitos relacionados a TB que foi registrado em 782 casos. Esses dados podem demonstrar uma fragilidade na notificação dessa patologia, o que é denominado de subnotificação, podendo estar relacionado com as lacunas no preenchimento dos registros (ESPINDOLA LCD, 2010). Ao longo do período em estudo ficou apontado que durante o ano de 2012 houve o maior número de mortalidade, com um total de 38,18 óbitos/ 100 mil habitantes e uma redução no ano de 2017 para 21,89/ 100 mil habitantes.

\section{CONCLUSÃO}

Conclui-se que mesmo com a grande incidência de casos por Tuberculose no Maranhão, houve uma queda gigantesca entre o início da pesquisa no ano de 2009 onde mostrava que grande parte da população nordestina sofria com a infecção até o ano de 2018, que como podemos observar nas análises teve uma redução bastante relevante. Foi mostrada também a forma infectante com maior predominância junto com o sexo, o qual foi definido como o masculino entre 20 e 39 anos por serem indivíduos mais expostos às condições de risco. Assim, faz-se necessário o incentivo da participação desses indivíduos, com mobilização e projetos que estimulem a participação e o seu envolvimento. Adicionalmente, espera-se que o projeto venha ajudar ao acompanhamento de estudos relacionados à Mycobacterium tuberculosis com dados recentes e atualizados em relação à doença.

\section{REFERÊNCIAS}

1. BARREIRA D e GRANGEIRO A. Avaliação das estratégias de controle da tuberculose no Brasil. Revista de Saúde Pública, 2007;41, 4-8.

2. BENTO J, et al. Métodos diagnósticos em tuberculose. Acta Médica Portuguesa, 2011; 24(1).

3. BERALDO AA, et al. Atraso na busca por serviço de saúde para o diagnóstico da tuberculose em Ribeirão Preto (SP). Ciência \& Saúde Coletiva, 2012; 17, 3079-3086.

4. BIERRENBACH AL, et al. Tendência da mortalidade por tuberculose no Brasil, 1980 a 2004. Revista de Saúde Pública, 2007; 41,15-23.

5. BRASIL. Ministério da Saúde. Secretaria de Vigilância em Saúde. Programa Nacional de Controle da Tuberulose. Situação da Tuberculose no Brasil. Brasília: Ministério da Saúde. 2012.

6. CEINFO. Análise do Quesito Raça/Cor a partir de Sistemas de Informação da Saúde do SUS". Boletim CEInfo Análise. Ano VI, oㅡ 05. São Paulo: Secretaria Municipal da Saúde, 2011. 54 p.

7. CHIRINOS NEC, MEIRELLES BHS. Fatores associados ao abandono do tratamento da tuberculose: uma revisão integrativa. Texto and Contexto Enfermagem, 2011; 20(3), 399.

8. CLEMENTINO FDS, et al. Tuberculose: desvendando conflitos pessoais e sociais. Rev. enferm. UERJ, 2011; 19(4), 638-643.

9. ESPINDOLA LCD. Estudo da mortalidade por tuberculose em Campo Grande - MS, 2001 a 2008 (Dissertação). Rio de Janeiro: Escola Nacional de Saúde Pública; 2010.

10. GROSCH CA, et al. Prevalência da tuberculose no Maranhão. Ver. Investig. Bioméd. São Luís. 2015; 7: 2834.

11. HINO P, et al. Revista Ciência \& Saúde Coletiva. 2011; 16: supl. 1, p. 1295-1301.

12. MENDES ADM, FENSTERSEIFER LM. Tuberculose: por que os pacientes abandonam o tratamento? Boletim de Pneumologia Sanitária, 2004;12(1), 27-38.

13. NETO MS, et al. Perfil clínico e epidemiológico e prevalência da coinfecção tuberculose/HIV em uma regional de saúde no Maranhão. J. Bras. Pneumol. 2012; 38(6): 724-732.

14. PEREIRA PR, et al. Desigualdades intraurbanas dos indicadores de saneamento em São Luís - MA [Internet]. São Luís: Universidade Federal do Maranhão; 2013.

15. PILLER RV. Epidemiologia da tuberculose. Pulmão RJ, 2012; 21(1), 4-9.

16. PONCE MAS, et al. Diagnóstico da tuberculose: desempenho do primeiro serviço de saúde procurado em São José do Rio Preto, São Paulo, Brasil. Cadernos de Saúde Pública, 29, 945-954.2013. 
17. RUFFINO-NETTO A. Tuberculose: a calamidade negligenciada. Rev Soc Bras Med Trop, 2002; 35(1), 51-8.

18. SAN PEDRO A, et al. Tuberculose e indicadores socioeconômicos: revisão sistemática da literatura. Revista Panamericana de Salud Pública, 2003; 33, 294-301.

19. SANTOS J. Resposta brasileira ao controle da tuberculose. Revista de Saúde Pública, 2007;41, 89-93.

20. SILVA IP, et al. Aspectos clínicos e epidemiológicos da tuberculose no município de Imperatriz, Maranhão. Enciclopédia Biosfera. Centro Científico Conhecer - Goiânia. 2016, 13(24): 1386.

21. WORLD HEALTH ORGANIZATION. Global tuberculosis report 2014. Geneva: WHO; 2013. 